\title{
Prosodic Feature Introduction and Emotion Incorporation in an Arabic TTS
}

\author{
O. Al-Dakkak ${ }^{(1)}$ and N. Ghneim ${ }^{(2)}$ \\ HIAST \\ P.O. Box 31983, Damascus, SYRIA \\ phone: $+(963-11) 512054$ \\ fax: + (963-11) 2237710. \\ (1)email: odakkak@hiast.edu.sy \\ (2)email:n_ghneim@netcourrier.com
}

\author{
M. Abou Zliekha ${ }^{(3)}$ and S. Al-Moubayed ${ }^{(4)}$ \\ Damascus University \\ Faculty of Information Technology \\ (3) email: mhd-it@scs-net.org \\ (4)email:kamal@scs-net.org
}

\begin{abstract}
Text-to-speech is a crucial part of many Man-Machine Communication applications, such as phone booking and banking, vocal e-mail, and many other applications. In addition to many other applications conceming impaired persons, such as: reading machines for blinds, talking machines for persons with speech difficulties. However, the main drawback of most speech synthesizers in the talking machines, are their metallic sounds. In order to sound naturally, we have to incorporate prosodic features, as close as possible to natural prosody, this helps to improve the quality of the synthetic speech. Actual researches in the world are towards better "Automatic Prosody Generation".
\end{abstract}

\section{Introduction}

With the objective of building a complete system of standard spoken Arabic with a high speech quality, we've built our Arabic Text-to-Speech. The input of our system is vocalized Arabic text, an expert system based on TOPH (Orthographic-PHonetic Transliteration) language [1], [2] transcripts the text into phonetic codes. In the actual version, we generate speech using the MBROLA diphones [3] (in a future version, semi-syllables will be used). MBROLA permits the control of some prosodic features (fundamental frequency F0, duration), which enabled us to construct our prosodic models and test it. In what follows, we discuss automatic prosody generation in our TTS (this enables the hearer to distinguish assertions, interrogations, exclamations), in addition to emotion inclusion in the synthesized speech.

\section{Arabic TTS}

To achieve our goals, we adopted the following steps (1) the definition of the phonemes' set used in standard Arabic, we have 38 phonemes, 28 consonants and 5 vowels $(/ \mathrm{a} /, / \mathrm{u} /, / \mathrm{i} /$ and the opened vowels $/ \mathrm{o} /$ and $/ \mathrm{e} /$ with 5 emphatic vowels, (2) the establishment of the Arabic text-to-phonemes rules using TOPH after its adaptation to Arabic Language, (3) the definition of the acoustic units; the semi-syllables, and the corpus from which these units are to be extracted.

As the Arabic syllables are only of 4 forms: $\mathrm{V}, \mathrm{CV}$, CVC, CVCC, the semi-syllables are of 5 forms: \#CV, $\mathrm{VC \#}, \mathrm{VCC \#}$ (\# is silence), and in continuous speech we have VCV and VCCV; hence the logatoms from which those semi-syllables are extracted are respectively [4], [5]: Crsasa, satVC, satVClC2, tV1CV2sa, $\mathrm{tV} 1 \mathrm{C} 1 \mathrm{C} 2 \mathrm{~V} 2 \mathrm{sa}$. Where the small letters are pronounced as they are, V, V1, V2 scans all the vowels and $\mathrm{C}, \mathrm{C} 1, \mathrm{C} 2$ scan all the consonants. Some combinations that never occur in the language are excluded (4) the recording process of the above corpus (not finished yet). It will be segmented and analyzed using PSOLA techniques [6], and in parallel (5) the incorporation of prosodic features in the syntactic speech (6) and the emotion inclusion in the TTS

\section{Automatic prosody generation}

When people read texts, they can differently read continuous sentences without stop, depending on their physiological and linguistic capabilities. The way people speak varies a lot according to the semantic of the uttered speech, the psychological state of the speaker, the situation, the emotion and other parameters. We recall works previously done in the field of general prosody generation for Arabic TTS, such as the ones in [7], [8].

In fact, semantically speaking, and according to linguists, sentences in Arabic are either informative (affirmative, negative and averment) or creative (requests, conditionals and special cases like: exclamation, swearing, ...) [9].

For our prosody generation, we will not use any semantic information. We will repose only on the punctuation to give the type of the sentence: Exclamation 
if it is ended by the exclamation point '!', Interrogative if it is ended by a question mark '?'. Continuous affirmation if ended by comma ',' and Affirmation (Long) if ended by a point '.' [10]

\section{3-1 The Corpus}

We began by a corpus recorded and studied in [9]. This corpus was composed of twelve short sentences of each of the four types (informative, interrogative, exclamation, ban), pronounced by five people four males and one female. Each sentence consists of four to fourteen phonemes. These sentences were analyzed and we have the curves for the fundamental frequency $\mathrm{F} 0$ and the intensity versus time.

A statistical study of these curves gave the general behavior of F0 and the intensity to have a first guess of these curves behaviors. F0 for all types of the sentences is increasing in the beginning, decreasing at the end, except for the interrogative sentences. The curves witness many peaks and valleys; the most important peak comes in the first syllable.

Then, a more detailed study showed that for the informative sentences the last decreasing of the sentence is very quick, the summit is on the second vowel (Ex. الجو باردد صباحا it's cold in the morning).

Concerning the interjections, the last part of F0 is either flat or decreasing, The summit is on the first vowel of the interjection tool L , F0 is in general greater than in the case of informative sentence (Ex. ما أروع عملك هذا! What a marvelous work!)

The interrogative sentences, F0 is high at the beginning and at the end of the sentence; the rest of the curve is either flat or ascending (Ex, هل ذابت انظلوج في Did the snow melt in spring?).

\section{3-2 Prosody Analysis}

In addition to this study, we chose other informative, interrogative and interjection sentences of variable lengths, and did the analysis of F0 using ten sentences of each of the four types (two types for informative sentences (continuous and final), interrogative, exclamation), pronounced by three speakers.

We noticed that according to the length of the sentence, we still have remarkable tendencies. We tried to group the sentences of each type, according to its length, in three groups: short (with a number of phonemes roughly between four and twenty phonemes), medium (between twenty and forty phonemes) and long (more than forty phonemes).

As it is not logic to provide a pattern for each sentence length, and the frontiers between the sets are not so rigid, we have used fuzzy sets to redefine the concept of the sentences' length. We therefore defined the following sets:

Short:

- The sentence is $100 \%$ short, if sent_length $<5$

- The sentence is not short if sent length $>20$

\section{Medium:}

- The sentence is $100 \%$ medium if $20<$ sent length $<30$

- The sentence is not medium if sent_length $<5$ or sent_length $>45$

Long:

- The sentence is $100 \%$ long if sent_length > 45

- The sentence is not long if sent_length $<30$

Consequently, we have the following curves of affiliations, shown in figure (1).

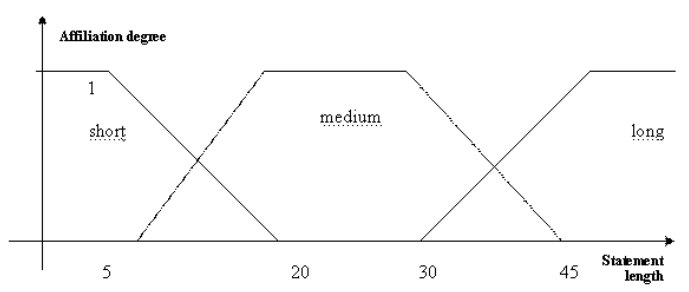

Figure 1. Affiliation of sentences to different fuzzy sets according to the length ( $\mathrm{nb}$. of phonemes)

\section{3-3 Prosody Generation}

According to the analysis results, we defined three patterns (corresponding to the classification of the sentence length: short, medium and long) of F0 for each of the four types of the adopted sentences. Each pattern is defined by values of $\mathrm{F} 0$, at interval of $5 \%$ or $10 \%$ of the sentence see table (1). The values are also given as percentages, a value of $0 \%$ corresponds to $70 \mathrm{~Hz}, 100 \%$ corresponds to $200 \mathrm{~Hz}$ (values corresponding to the locator of Arabic diphones taken from MBROLA).

We form three F0 curves of the synthesized sentence (one curve for each of the three adopted length: short, medium, long). As the sentences have not always the same number of phonemes, we make the necessary interpolations depending on the real length of the sentence.

The final synthesized $\mathrm{F} 0$ curve is a linear combination of the three resulting curves, depending on the degree of affiliation of the sentence length to each of the three length categories.

Table 1. Fo patterns for Medium Exclamation and Interrogation sentences. 


\begin{tabular}{|c|c|}
\hline Exclamation (Medium) & Interrogation (Medium) \\
\hline$<$ OFFSET $=\mathbf{5} \quad \mathrm{F} 0=\mathbf{0} />$ & $<$ OFFSET $=\mathbf{5}$ F0 $=\mathbf{8 0}>$ \\
\hline$<$ OFFSET $=10$ F0 $=10>$ & $<$ OFFSET $=10 \mathrm{~F} 0=\mathbf{1 0 0}>$ \\
\hline$<$ OFFSET $=\mathbf{1 5}$ F $0=\mathbf{2 0}>$ & $<$ OFFSET $=15$ F $0=90 />$ \\
\hline$<$ OFFSET $=\mathbf{2 0} \quad \mathrm{F} 0=\mathbf{3 5}>$ & $<$ OFFSET $=\mathbf{2 0} F 0=\mathbf{6 0}>$ \\
\hline$<$ OFFSET $=\mathbf{2 5}$ F $0=\mathbf{5 0}>$ & $<$ OFFSET $=\mathbf{2 5}$ F $0=\mathbf{2 0}>$ \\
\hline$<$ OFFSET $=\mathbf{3 0}$ F0 $0=\mathbf{5 2} \quad>$ & $<$ OFFSET $=\mathbf{3 5}$ F $0=\mathbf{3 0}>$ \\
\hline$<$ OFFSET $=\mathbf{4 0}$ F0 $=\mathbf{5 4}>$ & $<$ OFFSET $=45 \mathrm{~F} 0=\mathbf{4 0} />$ \\
\hline$<$ OFFSET $=\mathbf{5 0}$ F0 $=\mathbf{6 0}>>$ & $<$ OFFSET $=\mathbf{5 5}$ F0 $=\mathbf{5 0}>$ \\
\hline$<$ OFFSET $=\mathbf{5 5} \quad \mathrm{F} 0=75>$ & $<$ OFFSET $=65$ F0 $=60 />$ \\
\hline$<$ OFFSET $=65$ F0 $=85>$ & $<$ OFFSET $=75$ F $0=70>$ \\
\hline$<$ OFFSET $=75$ F0 $=60>$ & $<$ OFFSET $=\mathbf{8 5} \mathrm{F} 0=\mathbf{8 0}>$ \\
\hline$<$ OFFSET $=\mathbf{8 5}$ F0 $=\mathbf{4 0}>>$ & $<$ OFFSET $=95 \mathrm{~F} 0=90 />$ \\
\hline$<$ OFFSET $=\mathbf{9 5}$ F0 $=\mathbf{5 0}>$ & $<$ OFFSET $=\mathbf{1 0 0} \mathrm{F} 0=\mathbf{1 0 0}>$ \\
\hline$<$ OFFSET $=\mathbf{1 0 0} \mathrm{F} 0=\mathbf{0} \quad>$ & \\
\hline
\end{tabular}

In spite of the fact that this prosody generation is rather rough, it gave good results on the perception of the synthesized texts (see results below). An additional study is done concerning emotion inclusion in the TTS, using all the prosodic parameters: F0, intensity and duration. As we are actually using MBROLA acoustic diphones, and this application permits the control of $\mathrm{FO}$, and the duration but not the intensity, we built a tool to control the intensity on top MBROLA. This study is detailed in the following paragraph.

\section{Emotion inclusion in the TTS}

The most crucial acoustic parameters to consider for emotion synthesis are the prosodic parameters: pitch, duration and intensity [2], [3]. The variations of each of these parameters are described through the following other sub-parameters [2], [11], [12], [13]:

\section{F0 Parameter:}

- F0 Range (difference between F0max and F0min)

- Variability (degree of variability: high, low..)

- Average F0

- Contour slope (shape of contour slope)

- Jitter (irregularities between successive glottal pulses)

- Pitch variation according to phoneme class

\section{Duration Parameter}

- Speech rate

- Silence rate

- Duration variation according to phoneme class

- Duration variation according to pitch

Intensity Parameter

- Intensity variation according to pitch
Our methodology was to (1) record a corpus of sentences emotionless and with different emotions, (2) analyze these sentences to extract the various parameters and sub-parameters, and extract rules, (3) synthesize emotions according to these rules, and finally test the results and apply tuning on the rules when necessary.

\section{4-1 Recording, analysis and rules extraction}

Twenty sentences were chosen for each emotion. Each sentence was recorded twice, one emotionless and the other with the intended emotion. All these sentences were analyzed using PRAAT system to find the prosodic parameters. A statistical study followed to find the relevant changes between the pairs of sentences for each emotion. The following results were found (Table 2):

Table 2: Results on natural speech

\begin{tabular}{|c|c|}
\hline Emotion & Prosodic Rules \\
\hline Anger & $\begin{array}{l}\text { F0 mean: }+40 \%-75 \% \\
\text { F0 range: }+50 \%-100 \% \\
\text { F0 at vowels and semi-vowels: }+30 \% \\
\text { F0 slope: }+ \\
\text { Speech rate: + } \\
\text { Silence rate: - } \\
\text { Duration of vowels and semi-vowels: + } \\
\text { Intensity mean: + } \\
\text { Intensity monotonous with F0 } \\
\text { Others: F0 variability: }+, \mathrm{F} 0 \text { jitter: }+\end{array}$ \\
\hline joy & $\begin{array}{l}\text { F0 mean: }+30 \%-50 \% \\
\text { F0 range: }+50 \%-100 \% \\
\text { F0 at vowels and semi-vowels: }+30 \% \\
\text { F0 slope: - } \\
\text { Speech rate: - } \\
\text { Duration of vowels and semi-vowels: + } \\
\text { Intensity mean: }+ \\
\text { Intensity monotonous with F0 } \\
\text { Others: F0 variability: }+, \text { F0 jitter: }+\end{array}$ \\
\hline sadness & $\begin{array}{l}\text { F0 mean: }+40 \%-70 \% \\
\text { F0 range: }+180 \%-220 \% \\
\text { F0 at vowels and semi-vowels: }+ \\
\text { Speech rate: - } \\
\text { Silence rate: }+ \\
\text { Duration of vowels and semi-vowels: }+ \\
\text { Intensity mean: }+\end{array}$ \\
\hline fear & $\begin{array}{l}\text { F0 mean: }+50 \%-100 \% \\
\text { F0 range: }+100 \%-150 \% \\
\text { F0 at vowels, semi-vowels, nasals and } \\
\text { fricatives: }+ \\
\text { Speech rate: }+ \\
\text { Silence rate: - } \\
\text { Duration of vowels and semi-vowels: }+ \\
\text { Intensity mean: }+ \\
\text { Intensity monotonous with F0 }\end{array}$ \\
\hline
\end{tabular}




\begin{tabular}{|l|l|}
\hline & Others: F0 variability: +, F0 jitter: + \\
\hline surprise & F0 mean: $+50 \%-80 \%$ \\
& F0 range: $+150 \%-200 \%$ \\
& F0 at vowels and semi-vowels: + \\
& Speech rate: + \\
& Silence rate: - \\
& Duration of vowels and semi-vowels: + \\
& Others: F0 variability: + \\
\hline
\end{tabular}

\section{Emotion synthesis}

To test the above rules, we have developed a tool linked to our TTS system, to control emotional parameters over the Arabic text automatically. The inherent synthetic prosody (emotionless), built in the system is rather coarse, thus the application of the above rules did not give always the desired emotion perception. We had to tune those rules to cope with the synthesizer. The final experimental emotional rules are given below (Table 3):

Table 3: Results on synthetic speech

\begin{tabular}{|c|c|}
\hline Emotion & Prosodic Rules \\
\hline Anger & $\begin{array}{l}\text { F0 mean: }+30 \% \\
\text { F0 range: }+30 \% \\
\text { F0 at vowels and semi-vowels: }+100 \% \\
\text { Speech rate: }+75 \%-80 \% \\
\text { Duration of vowels and semi-vowels: }+30 \% \\
\text { Duration of fricatives: }+20 \%\end{array}$ \\
\hline joy & $\begin{array}{l}\text { F0 mean: }+50 \% \\
\text { F0 range: }+50 \% \\
\text { F0 at vowels and semi-vowels: }+30 \% \\
\text { F0 at fricative: }+30 \% \\
\text { Speech rate: }+75 \%-80 \% \\
\text { Duration of vowels and semi-vowels: }+30 \% \\
\text { Duration of last vowel phonemes: }+20 \% \\
\text { Others: F0 variability: }+40 \%\end{array}$ \\
\hline sadness & $\begin{array}{l}\text { F0 range: }+130 \% \\
\text { F0 at vowels and semi-vowels: }+120 \% \\
\text { F0 at fricative: }+120 \% \\
\text { Speech rate: }-130 \%\end{array}$ \\
\hline fear & $\begin{array}{l}\text { F0 mean: }+40 \% \\
\text { F0 range: }+40 \% \\
\text { F0 at vowels, semi-vowels, nasals and } \\
\text { fricatives: }+30 \% \\
\text { Speech rate: }-75 \%-80 \% \\
\text { Others: } \text { F0 variability: }+60 \% \text {, F0 jitter: }+3 \%\end{array}$ \\
\hline surprise & $\begin{array}{l}\text { F0 mean: }+220 \% \\
\text { F0 at vowels and semi-vowels: }+150 \% \\
\text { Speech rate: }-110 \% \\
\text { Duration of vowels: }+200 \% \\
\text { Duration of semi-vowels: }+150 \% \\
\text { Others: F0 variability: }+60 \%\end{array}$ \\
\hline
\end{tabular}

The following five figures show F0 contours for each emotional type sentence with its corresponding emotionless sentence.

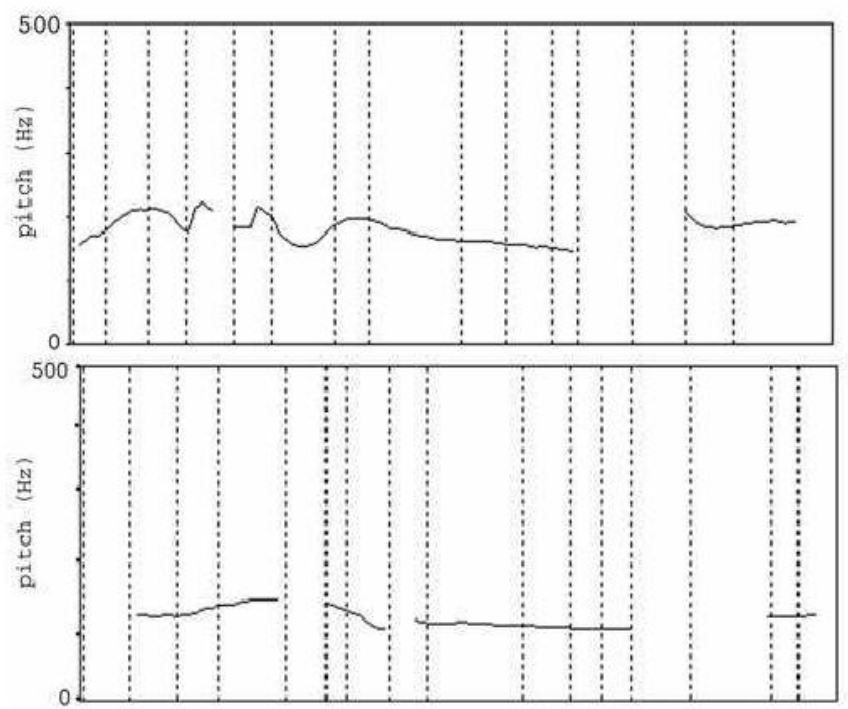

Figure 2: Anger emotion and emotionless / من تظن نفسك؟) "who do you think you are?"
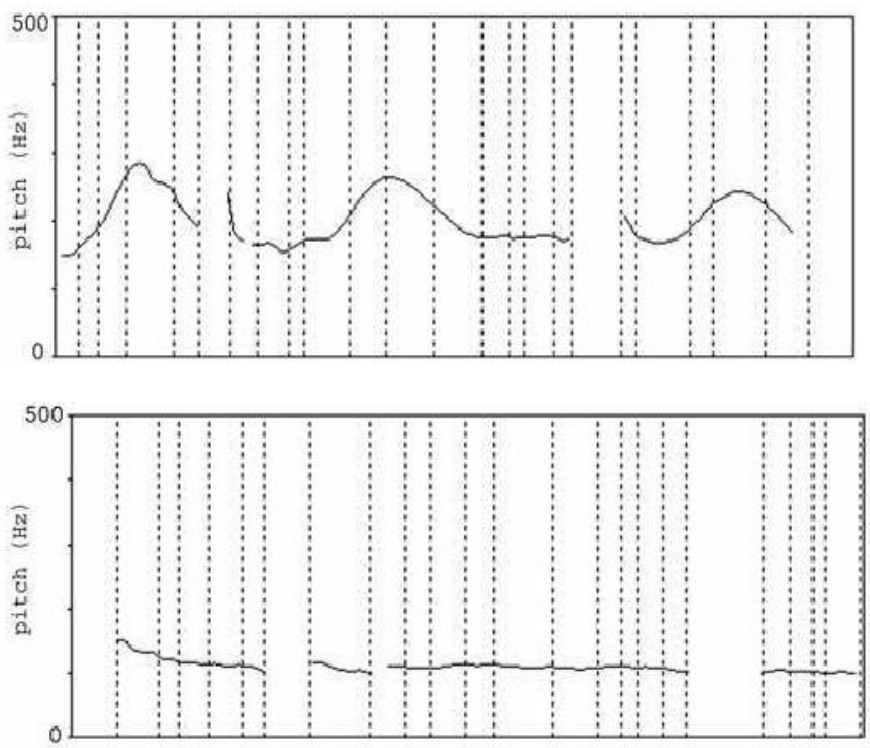

Figure 3: Joy emotion and emotionless / الت الفيوم من /لالسماء/ "No more clouds in the sky"

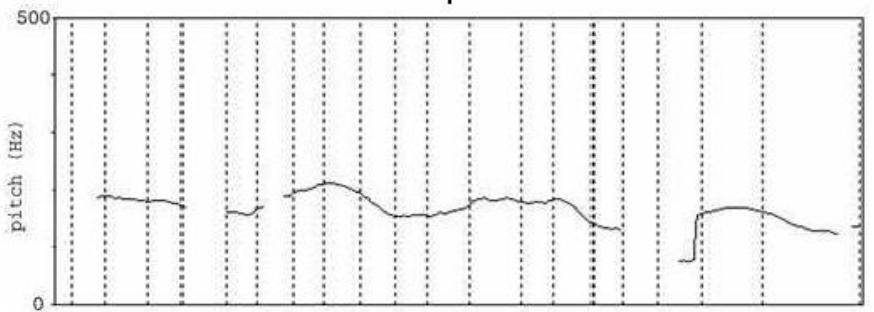




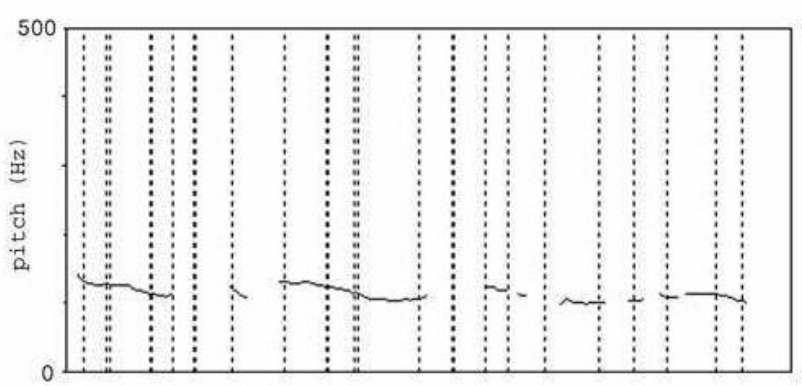

Figure 4: Sadness emotion and emotionless / أنا حزبين جدا : اليو/' "I am so sad today!"
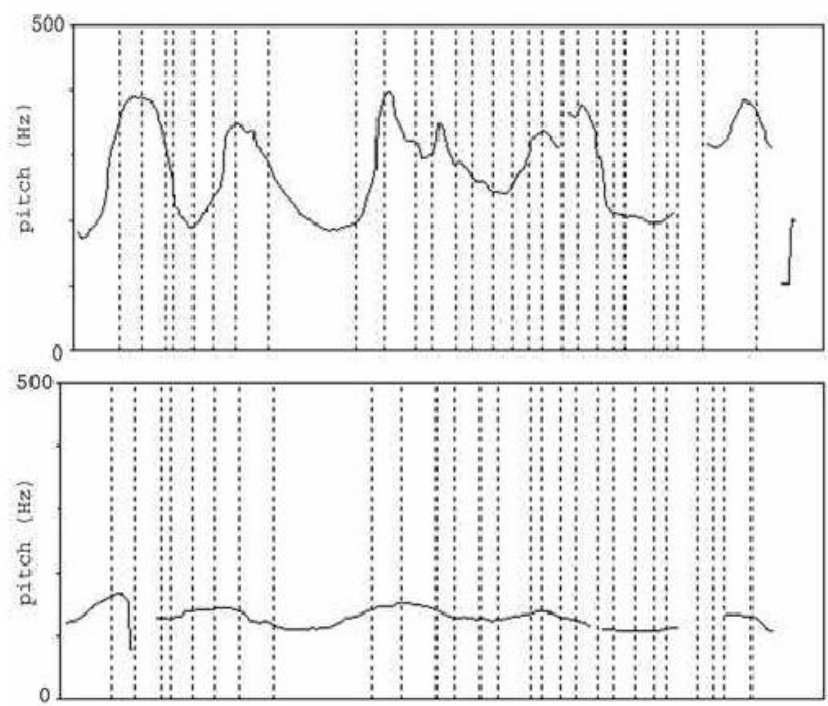

Figure 5: Fear emotion and emotionless إلهي ما هذا المنظر /لمخيف/"God! What a scary scene!"
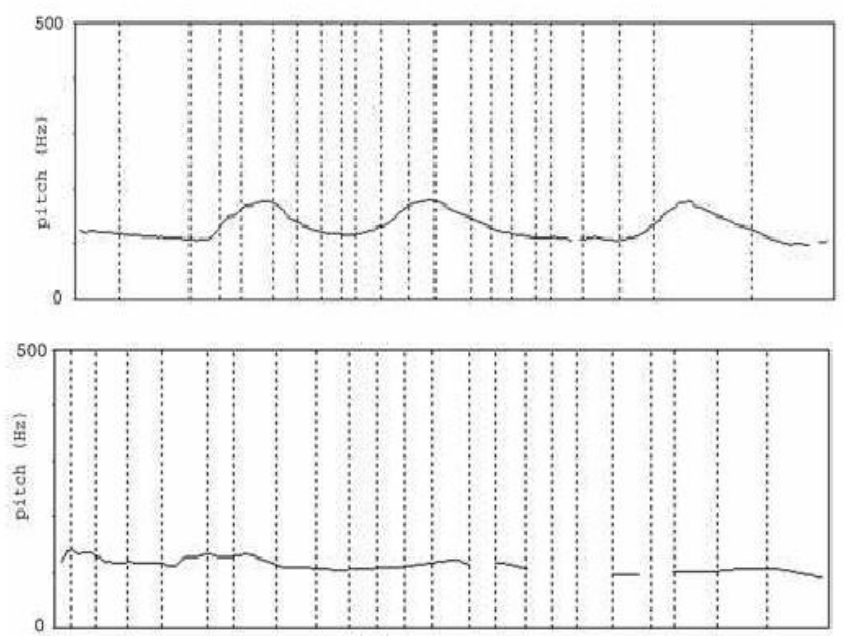

Figure 6: Surprise emotion and emotionless اله له من منظر جميل!"What a beautiful scene!"

\section{Results}

We performed a number of experiments, testing the rough prosody generation. We composed a set of three sentences of increasing length, for each of the three types of prosody (interrogation, exclamation and informative prosody). We synthesized all of the nine sentences by each of the prosody types; this means that we synthesized the interrogative sentences by interrogative intonation, then by exclamative intonation and finally by informative intonation. We let five listeners listen to the sentences for each synthesized prosody and tell the perceived prosody. The results are shown in the table (4)

Table 4: Prosody recognition for different types of sentences semantics.

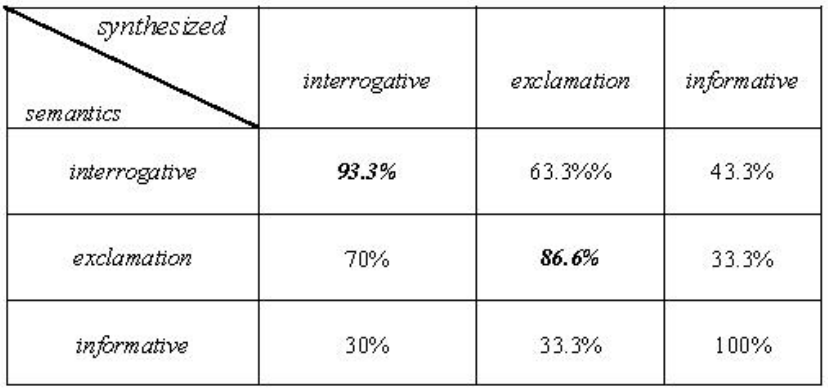

In fact, each cell of the above table is the results of a different experiment; a high rate means a high recognition rate of prosody. We notice that when we put interrogative intonation on question, we perceive interrogation in $93.3 \%$ of cases (in fact the prosody recognition rate is $100 \%$ in short sentences, and decreases with the length of the sentence). If we put interrogation intonation on other kinds of sentences, we notice that in $70 \%$ of exclamation cases we still recognize the interrogative intonation as interrogative, while with simple informative sentences we recognize poorly the interrogative intonation. Similar explanation can be given for the other two columns.

We can also notice that informative intonation prosody synthesized is $100 \%$ recognized on informative sentences whatever the length of the sentences is. Exclamation intonation prosody is recognized in about $87 \%$ of cases of sentences having exclamation semantics. This rate is lower on medium length sentences than short or long ones.

As the prosody recognition rates are fairly good according to the sentence semantics. We proceeded to emotion synthesis. As mentioned above, we worked with all the prosodic parameters: F0, duration and amplitude.

Using the experimental rules, five sentences for each emotion were synthesized and listened by 10 people. Each individual was asked to give the perceived emotion for each sentence. Table 5 shows the results of this test [Eusipco 2005]. 
Table 5: Emotion recognition rates.

\begin{tabular}{|c|l|l|l|l|l|l|}
\hline synthesized & Anger & Joy & Sadness & Fear & $\begin{array}{c}\text { Surpr } \\
\text { ise }\end{array}$ & Others \\
\hline Anger & $\mathbf{7 5 \%}$ & $0 \%$ & $2 \%$ & $7 \%$ & $0 \%$ & $6 \%$ \\
\hline Joy & $0 \%$ & $\mathbf{6 7 \%}$ & $0 \%$ & $2 \%$ & $13 \%$ & $18 \%$ \\
\hline Sadness & $5 \%$ & $0 \%$ & $70 \%$ & $5 \%$ & $0 \%$ & $20 \%$ \\
\hline Fear & $3 \%$ & $0 \%$ & $5 \%$ & $\mathbf{8 0 \%}$ & $0 \%$ & $12 \%$ \\
\hline Surprise & $0 \%$ & $10 \%$ & $0 \%$ & $2 \%$ & $\mathbf{7 3} \%$ & $15 \%$ \\
\hline
\end{tabular}

Emotion recognition of synthesized speech rates range from $67 \%$ to $80 \%$. Some people believed that some tested sentences have more than one emotion.

\section{Conclusion}

An automated tool has been developed for emotional Arabic synthesis. It is based on an automatic prosody rough generation model, which performs intonation modification taking into account some punctuation marks (', ' ', '!', and '?') and the number of phonemes in the sentence, using predefined patterns for different sentence lengths. The affiliation of the sentence to each pattern is governed by fuzzy logic. On top of this prosody, another prosody is built to synthesize different emotions.

The resulting prosodic model, proposed and tested in this work proved to be successful, especially when applied in conversational contexts.

A further work will follow to incorporate other emotions like disgust, and annoyance.

As the departing prosody plays a crucial role in emotion synthesis, we intend to refine our rough prosodic model to improve the quality of the TTS System; the emotional rules have to be revalidated to cope with it.

\section{References}

[1] O. Al dakkak, N. Ghneim, "Towards Man-Machine Communication in Arabic" in Proc. Syrian-Lebanese Conference, Damascus SYRIA, October 12-13, 1999.

[2] V. Aubergé, "La Synthèse de La parole: des Règles aux Lexiques", Thèse de l'université Pierre Mendès France, Grenoble2, 1991.

[3] T. Dutoit, V. Pagel, N. Pierret, F. Bataille and O. van der Vrecken,'The MBROLA project: towards a set of high quality speech synthesizers free of use for non-commercial purposes", Proc. of ICSLP'96, pp. 1393-1396, 1996.

[4] N. Chenfour, A. Benabbou and A. Mouradi, "Etude et Evaluation de la di-syllabe comme Unité Acoustique pour le Système de Synthèse Arabe PARADIS", Second International Conference on language resources and evaluation, Athenes, Greece, 31 May-2 June 2000.

[5] N. Chenfour, A. Benabbou and A. Mouradi, "Synthèse de la Parole Arabe TD-PSOLA Géneration et Codage Automatiques du Dictionnaire ", Second International Conference on language resources and evaluation, Athenes, Greece, 31 May-2 June 2000.

[6] E. Moulines and J. Laroche, "Non-parametric techniques for pitch-scale and time-scale modification of speech" Speech Communication, vol. 16, pp. 175-205, 1995.

[7] S. Nasser Eldin, H. Abdel Nour and A. Rajouani "Enhancement of a TTS System for Arabic Concatenative Synthesis by Introducing a Prosodic Model", ACL/EACL 2001 workshop, Toulouse-France 2001.

[8] S. Baloul, M. Alissali, M. Baudry and P. Boula de Mareuil "Interface syntaxe-prosodie dans un système de synthèse de la parole à partir du texte en arabe", XXIViemes Journées d'Etudes sur la Parole, CNRS/Université Nancy2, Nancy, France, 24-27 juin 2002.

[9] M. Albawab et al., finding intonation rules for some sentences in view of standard Arabic speech synthesis and recognition, internal report, HIAST, 1985

[10] H. Mashalah, and H. Trtrian, Text reader project, internal report, IT faculty, Damascus University, 2003

[11] F. Zotter, "Emotional speech", at URL: http://spsc.inw.tugraz.at/courses/asp/ws03/talks/zotter.pdf

[12] I. R. Murray, M. D. Edgington, D. Campion, and J. Lynn, "Rule-based emotion synthesis using concatenated speech", ISCA Workshop on Speech \& Emotion, Northern Ireland, 2000, pp. 173-177.

[13] J.M. Montero, J. Gutiérrez-Arriola, J. Colás, E.Enríquez and J.M.Pardo, "Analysis and modelling of emotional speech in Spanich", at URL: http://orien.die.upm.es/ juancho/conferences/0237.pdf

[14] O. Al-Dakkak, N. Ghneim, M. Abou-Zliekha and S. AlMoubayed, "Emotion Inclusion in an Arabic Text-to-Speech", Eusipco 2005, Turkey. 